\title{
CORRESPONDENCE
}

\section{Large-scale mapping of the Ebola NP and GP proteins reveals multiple immunoprevalent and conserved CD4 T-cell epitopes}

\author{
Yann Gallais ${ }^{1}$, Raphaël Sierocki ${ }^{1}$, Gautier Lhomme ${ }^{1}$, Coline Sivelle ${ }^{1}$, Divor Kiseljak ${ }^{2}$, Florian Wurm ${ }^{2}$, Sami Djoulah ${ }^{3}$, Ahmed Bouzidi ${ }^{4}$
} Jérôme Kerzerho ${ }^{4}$ and Bernard Maillère (iD) ${ }^{1}$

Cellular \& Molecular Immunology (2021) 18:1323-1325; https://doi.org/10.1038/s41423-020-0455-2

Humankind faces regular outbreaks from emerging viruses, such as Ebola and the current SARS-CoV-2, or from viruses circulating at low levels in the wild animal reservoir. Owing to the suddenness of virus expansion, the lack of knowledge of immune determinants and correlates of protection severely limit the ability to address the outbreaks. Identified in 1976, Ebola virus is part of the Filoviridae family and causes severe hemorrhagic fever with a mortality rate between 50\% and 90\%. From 2013 to 2020, more than 30,000 infections and 15,000 deaths have been reported worldwide. High antibody titers against Ebola virus GP are found in patients recovering from Ebola virus infection and correlate with protection in vaccination models. ${ }^{1}$ Both CD8 and CD4 T-cell responses also seem to participate in the control of Ebola virus infection in animals, and CD4 T cells are necessary to induce resistance to Ebola virus challenge. ${ }^{2}$ In addition to GP, NP induces strong T-cell responses in animal models that protect against Ebola virus infection, and in convalescent Ebola patients, most of the T-cell responses mounted against Ebola virus were found to target NP. ${ }^{3}$ However, very few T-cell epitopes specific for both the Ebola GP and NP proteins have yet been identified in humans. ${ }^{4,5}$ Only one study reported CD4 T-cell epitopes restricted to HLADR3. ${ }^{6}$ We therefore established a large-scale approach to identify CD4 T-cell epitopes using donors not exposed to infection to anticipate the sudden rise of emerging viruses and applied this approach to the Ebola GP and NP proteins.

Because the amplitude of the memory $\mathrm{T}$-cell response relies on the size of the naive T-cell repertoire, we used healthy donors as a source of T cells to identify CD4 T-cell epitopes. We compensated for the very low number of specific $T$ cells by generating specific T-cell lines. CD4 $\mathrm{T}$ cells collected from healthy donors were stimulated in 30 replicates by 4 weekly rounds of stimulation with pools of Zaire Ebola-derived GP and NP peptides, and their specificity was assessed by IFN- $\gamma$ ELISpot assays using peptide pools and individual peptides. Twenty-seven NP and 33 GP 20-mer peptides composed the peptide pools. They were selected with two public algorithms, NetMHCpan and Sturniolo, for their ability to bind to 15 HLA-DR molecules that are predominant in African and European populations but are also very common worldwide. Sixteen healthy donors with unrelated HLA typing were used to generate T-cell lines specific for Ebola peptides. As an example (Fig. S1), 123 T-cell lines from a single donor were found to be specific for either 23 NP or 12 GP peptides. A total of 979 specific T-cell lines were derived from all donors; $510 \mathrm{~T}$-cell lines were specific to NP peptides (Fig. 1a and Table S1) and 469 to GP peptides (Fig. $1 \mathrm{~b}$ and Table S1). Fifty-nine of the 60 predicted peptides induced a T-cell response in at least one donor, illustrating the efficiency of the prediction software. However, the number of specific T-cell lines and of responding donors was highly variable across the peptides. These discrepancies result from the large variability in the size of the epitope-specific T-cell repertoire and highlight the importance of the naive T-cell repertoire to shape the T-cell response.,

Ten NP and eight GP peptides generated specific T-cell lines in at least $50 \%$ of the tested donors. Interestingly, only a combination of four peptides (three from NP and one from GP) suffices to induce a T-cell response in all donors (Fig. S2) but corresponds to only $15 \%$ of the total response. Alternatively, a combination of 18 peptides accounted for more than $50 \%$ of the total response (Fig. S2). We therefore extended the characterization of the T cells raised against these peptides. Using HLA-specific antibodies, we found that most of the $18 \mathrm{~T}$-cell epitopes are restricted to HLA-DR molecules, as predicted in silico (Fig. 1c-e). Eight peptides were restricted at least partly to HLA-DP molecules, which shared HLADR common anchor residues, with the peptide NP80-99 being restricted to HLA-DP only. We also assessed the T-cell crossreactivity for the other Ebola strains (Fig. 1c-e). The initial peptide sequences were from the Ebola Zaire strain, as this strain is the first one reported and is among the most virulent strains. Two other Ebola strains lethal to humans have been identified (Sudan and Bundibugyo), with the Reston strain being nonlethal in humans, and there is only one nonfatal case reported (1994) with the Tai Forest strain (not considered here). The specificity of T-cell lines raised against Zaire epitopes was evaluated with corresponding Sudan, Reston, and Bundibugyo peptides by IFN- $\gamma$ ELISpot (Fig. 1c-e). Examples of partial and full cross-reactivity are shown in Fig. 1c and Fig. 1d, respectively. In addition to three NP peptides, which were completely conserved across the Ebola strains, one NP peptide was completely conserved between the Sudan and Reston strains and presented $86 \%$ cross-reactivity with Bundibugyo. Three NP peptides and two GP peptides exhibited a cross-reactivity greater than $66 \%$ to Sudan-derived peptides, which is the second most virulent Ebola strain. Another peptide cross-reacted with the other strains, while 2 peptides crossreacted with Bundibugyo only. Variants of three GP epitopes were not tested because they were too divergent from their Zaire strain counterparts. We also investigated whether the identified T-cell epitopes were immunodominant and hence were naturally processed from the whole Ebola proteins and presented to

\footnotetext{
${ }^{1}$ Département Médicament et Technologie pour la Santé, SIMoS, Université Paris-Saclay, CEA-Saclay, 91191 Gif surYvette, France; ${ }^{2}$ Excellgene, Monthey, Switzerland; ${ }^{3}$ Wiratech Europe, Genopole, 91000 Evry, France and ${ }^{4}$ Vaxeal holding SA, Vevey, Switzerland

Correspondence: Bernard Maillère (bernard.maillere@cea.fr)
}

Received: 14 April 2020 Accepted: 18 April 2020

Published online: 12 May 2020 
A

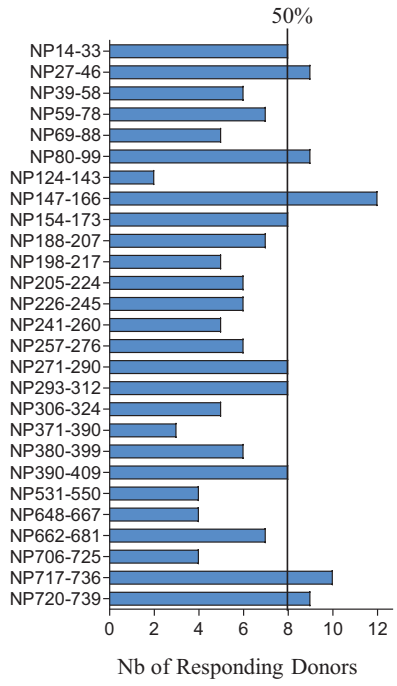

$\mathrm{C}$

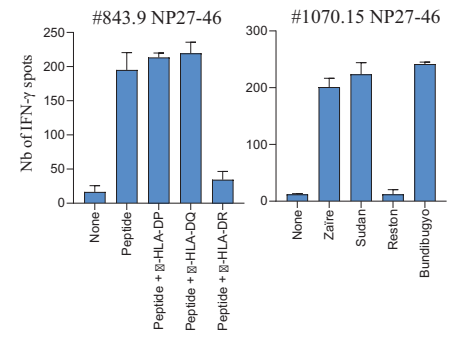

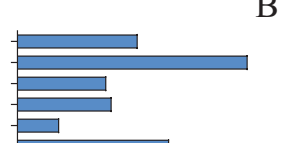

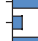

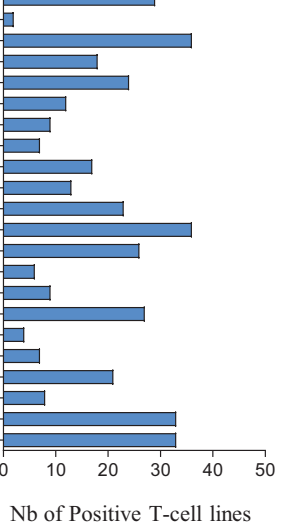

B

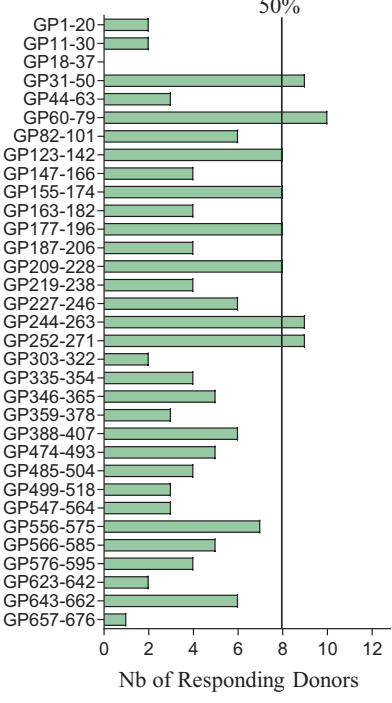

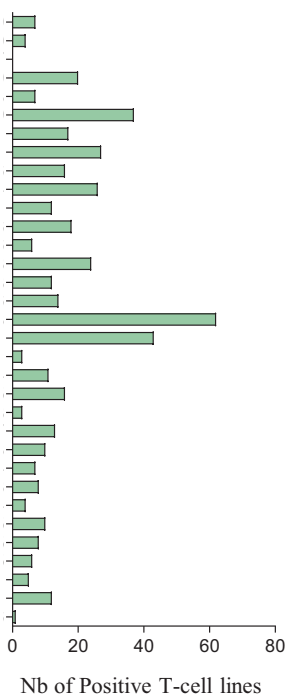

$\mathrm{D}$
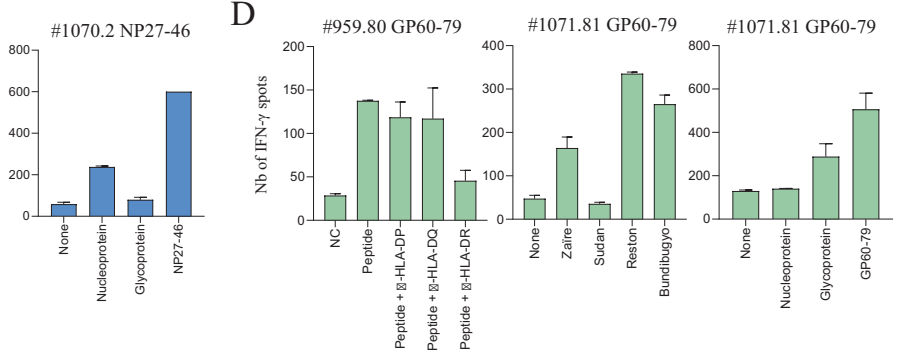

$\mathrm{E}$

\begin{tabular}{cccccccccc}
\hline Peptide & $\begin{array}{c}\text { Responding } \\
\text { Donors } \\
(\%)\end{array}$ & $\begin{array}{c}\text { Intensity } \\
(\%)\end{array}$ & Sudan & Reston & Bundibugyo & DP & DQ & DR & $\begin{array}{c}\text { Specificity for } \\
\text { protein }\end{array}$ \\
\hline NP14-33 & 50 & 4,5 & 67 & 100 & 100 & - & - & 100 & + \\
NP27-46 & 56 & 8,6 & 91 & 4 & 87 & - & - & 100 & + \\
NP80-99 & 56 & 5,7 & 13 & 0 & 0 & 100 & - & - & + \\
NP147-166 & 75 & 7,1 & id. & id. & id. & 40 & - & 60 & + \\
NP154-173 & 50 & 3,5 & id. & id. & id. & 57 & - & 43 & + \\
NP271-290 & 50 & 4,5 & 67 & 44 & 78 & 33 & - & 66 & + \\
NP293-312 & 50 & 7,1 & id. & id. & id. & - & - & 100 & + \\
NP390-409 & 50 & 5,3 & id. & id. & 86 & - & - & 100 & - \\
NP717-736 & 62 & 6,5 & 8 & 15 & 69 & 30 & - & 70 & + \\
NP720-739 & 56 & 6,5 & 0 & 10 & 60 & 50 & - & 50 & + \\
\hline GP31-50 & 56 & 4,3 & 0 & 17 & 17 & - & - & 100 & + \\
GP60-79 & 63 & 7,9 & 38 & 75 & 75 & - & - & 100 & + \\
GP123-142 & 50 & 5,8 & 73 & 73 & 100 & - & - & 100 & + \\
GP155-174 & 50 & 5,5 & 70 & 80 & 90 & - & - & 100 & + \\
GP177-196 & 50 & 3,8 & nt & nt & nt & 50 & - & 50 & + \\
GP209-228 & 50 & 5,1 & nt & nt & nt & 50 & - & 50 & + \\
GP244-263 & 56 & 13,2 & 0 & 44 & 75 & - & - & 100 & + \\
GP252-271 & 56 & 9,2 & nt & nt & nt & - & - & 100 & + \\
\hline
\end{tabular}

Fig. 1 Mapping and characterization of CD4 T-cell responses to NP and GP from Zaire Ebola virus. Peptide-specific T-cell lines were generated by 4 weekly rounds of stimulation of immunopurified CD4 T cells collected from 16 healthy donors, with 4 peptide pools from the NP (a) and GP (b) proteins loaded on autologous PBMCs. Each pool contained 15 peptides. CD4 T-cell specificity was analyzed by IFN- $\gamma$ ELISpot assays using peptide pools in a first assay and individual peptides from these pools in a second assay. CD4 T-cell lines were considered specific when a spot count was twofold higher than that in the absence of peptides, with a minimal difference of 25 spots. Examples of CD4 T-cell lines specific to (c) NP peptides and to (d) GP peptides. (e) Summary of data obtained for the 18 selected peptides. HLA restriction was assessed using monoclonal antibodies specific to HLA-DP (B7/21), HLA-DQ (SPVL3) or HLA-DR (L243). Each antibody was introduced into the IFN- $\gamma$ ELISpot assay at a final concentration of $10 \mu \mathrm{g} / \mathrm{ml}$. Restriction was confirmed when inhibition was higher than $50 \%$ of the ELISpot count with the peptide only. Cross-reactivity of T-cell lines was investigated with peptides from Sudan, Reston, and Bundibugyo strains. id.: identical sequence between the variant and Zaire strain, nt: not tested due to high divergence in the variant sequence compared to that in the Zaire strain. Recognition of the naturally processed epitopes was assessed using autologous DCs loaded with $3 \mu \mathrm{M}$ recombinant NP or GP proteins, with each protein being a control for the other protein 
Large-scale mapping of the Ebola NP and GP proteins reveals multiple... Y Gallais et al.

T cells. Peptide-specific T-cell lines were tested for their capacity to be activated by dendritic cells loaded with either the recombinant NP or GP Ebola protein (Fig. 1c-e). Aside from those recognizing the peptide NP390-409, all the peptide-specific T-cell lines were stimulated by either the recombinant NP or GP protein. TCR VBeta repertoire analysis of 23 T-cell lines specific for 3 NP peptides (NP27-46, NP80-99 and NP147-166) and 8 T-cell lines specific for 3 GP peptides (GP31-50, GP60-79 and GP123-142) from 3 different donors was conducted, showing that the T-cell response was shaped mainly by the individual repertoire of the donor (Table S2).

In this study, we therefore identified 17 immunodominant CD4 T-cell epitopes from the Ebola Zaire NP and GP proteins recapitulating approximately half of the magnitude of the T-cell response and generating a $T$ cell response in all the tested donors. These epitopes are either fully conserved or induce cross-reactive T-cell responses with their counterparts from other Ebola strains. These 17 T-cell epitopes thus appear to be the most important CD4 T-cell epitopes to the Ebola T-cell response. Although vaccine candidates contain only GP as an Ebola component, we revealed here multiple T-cell epitopes from the NP protein, 9 of them being part of the most important 17 CD4 T-cell epitopes we identified. These epitopes are either fully conserved or induce a crossreactive T-cell response. NP appears therefore as a relevant target to elicit a strong CD4 T-cell response to Ebola virus and thus could be included in the design of new Ebola vaccines. Our approach relies on the use of CD4 T cells collected from healthy donors. In addition to the advantage of obtaining a large number of $T$ cells, its main strength resides in its faculty to anticipate the T-cell response before any infection, valuable information in the current era of the emergence of COVID-19.

\section{ACKNOWLEDGEMENTS}

The research leading to these results was supported by the Innovative Medicines Initiative Joint Undertaking PEVIA project under grant agreement \#116088, the resources of which comprise financial contributions from the European Union. The authors thank Tiphanie Pruvost and Evelyne Correia from SIMoS for helpful discussions and technical advice.

\section{AUTHOR CONTRIBUTIONS}

Y.G., B.M., A.B, J.K., S.D., and F.W. designed the experiments; Y.G., R.S., G.L., C.S, and D. K. performed the experiments; Y.G., G.L., and B.M. analyzed the data; and Y.G. and B. M. wrote the paper.

\section{ADDITIONAL INFORMATION}

The online version of this article (https://doi.org/10.1038/s41423-020-0455-2) contains supplementary material.

Competing interests: Y.G. and B.M. are inventors of a pending patent.

\section{REFERENCES}

1. Wong, G., Kobinger, G. P. \& Qiu, X. Characterization of host immune responses in Ebola virus infections. Expert Rev. Clin. Immunol. 10, 781-790 (2014).

2. Rao, M., Bray, M., Alving, C. R., Jahrling, P. \& Matyas, G. R. Induction of immune responses in mice and monkeys to Ebola virus after immunization with liposomeencapsulated irradiated Ebola virus: protection in mice requires CD4+ T cells. J. Virol. 76, 9176-9185 (2002).

3. McElroy, A. K. et al. Human Ebola virus infection results in substantial immune activation. Proc. Natl Acad. Sci. USA 112, 4719-4724 (2015).

4. Ruibal, P. et al. Unique human immune signature of Ebola virus disease in Guinea. Nature 533, 100-104 (2016).

5. Sakabe, S. et al. Analysis of CD8+ T cell response during the 2013-2016 Ebola epidemic in West Africa. Proc. Natl Acad. Sci. USA 115, E7578-E7586 (2018).

6. Bounds, C. E. et al. An immunoinformatics-derived DNA vaccine encoding human class II T cell epitopes of Ebola virus, Sudan virus, and Venezuelan equine encephalitis virus is immunogenic in HLA transgenic mice. Hum. Vaccines Immunother. 13, 2824-2836 (2017).

7. Kwok, W. W. et al. Frequency of epitope-specific naive CD4+ T cells correlates with immunodominance in the human memory repertoire. J. Immunol. 188, 2537-2544 (2012).

8. Moon, J. J. et al. Naive CD4+ T cell frequency varies for different epitopes and predicts repertoire diversity and response magnitude. Immunity 27, 203-213 (2007). 